\title{
Doctors' experiences of adverse events in secondary care: the professional and personal impact
}

\author{
Authors: Reema Harrison, ${ }^{A}$ Rebecca Lawton ${ }^{B}$ and Kevin Stewart ${ }^{C}$
}

\begin{abstract}
We carried out a cross-sectional online survey of fellows and members of the Royal College of Physicians to establish physicians' experiences of adverse patient safety events and near misses, and the professional and personal impact of these. 1,755 physicians answered at least one question; 1,334 answered every relevant question. Of 1,463 doctors whose patients had an adverse event or near miss, 1,119 (76\%) believed this had affected them personally or professionally. $1,077(74 \%)$ reported stress, 995 (68\%) anxiety, $840(60 \%)$ sleep disturbance and 886 (63\%) lower professional confidence. 1,192 (81\%) became anxious about the potential for future errors. Of 1,141 who had used NHS incident reporting systems, only 315 (28\%) were satisfied with this process. 201 (14\%) received useful feedback, 201 (19\%) saw local improvements and 277 (19\%) saw system changes. 364 (25\%) did not report an incident that they should have. Adverse safety events affect physicians, but few formal sources of support are available. Most doctors use incident-reporting systems, but many describe a lack of useful feedback, systems change or local improvement.
\end{abstract}

KEYWORDS: $x x x$.

\section{Introduction}

In the wake of recent high-profile quality failures, the safety of NHS patients is of widespread concern. ${ }^{1,2}$ Despite significant investment in incident-reporting systems, as well as professional and regulatory requirements to support their use, rates of adverse event reporting are low, particularly amongst doctors. ${ }^{3-10}$ Many clinicians are also reluctant to disclose details of adverse events (see Box 1) to patients and their families. ${ }^{11,12}$

Multiple factors are thought to contribute to this, including the psychological effects on clinicians of involvement in adverse patient safety events, a fear by them that their organisation will take a punitive approach to any investigation, and a lack of confidence that systems will change as a result of reporting.

Authors: A research fellow, Sydney Medical School, University of Sydney, Sydney, Australia; ${ }^{B}$ professor of the psychology of healthcare, Institute of Psychological Sciences, University of Leeds, Leeds, UK; ' Clinical director, Royal College of Physicians' Clinical Effectiveness and Evaluation Unit, London, UK.

\section{Box 1. Definition of an adverse event and a near miss.}

An adverse event describes 'an injury related to medical management, in contrast to complications of disease', whereas a 'near miss' describes a 'serious error or mishap that has the potential to cause an adverse event but fails to do so because of chance or because it is intercepted. ${ }^{38}$

Negative experience of previous incident investigations may reinforce these concerns. ${ }^{13-18}$

There is a growing body of evidence to suggest that clinicians who directly or indirectly contribute to the occurrence of an adverse event can experience psychological effects that disrupt their professional and personal lives, as well as their ability to deliver high-quality, safe care. ${ }^{18-23}$ Anxiety, depression, sleep disturbance, fear and worry are consistently reported by those involved in adverse events, as are shame, guilt, loss of selfconfidence, and feelings of incompetence and worthlessness. ${ }^{23-29}$ The severity of these effects is related to the degree of harm to the patient and the clinician's experience of the investigation process; they are more pronounced with more serious incidents. ${ }^{30,31}$

These effects have adverse consequences for patients, for clinicians and for the wider NHS. Patient safety is at risk in the immediate aftermath of an incident, when a clinician's ability to manage other patients may be impaired. ${ }^{26}$ In days and weeks following an incident, stress, anxiety and sleep disturbance may affect clinical decision making, job performance and colleague relationships. In the longer term, safety culture and the ability to learn from adverse events is threatened if clinicians are reluctant to report incidents and transparency is supressed. ${ }^{26}$ In extreme cases, clinicians may consider changing career or leaving the profession. ${ }^{26,32}$

Most reports of this phenomenon are from the United States, where several programmes have been established to support clinicians who are affected. ${ }^{26,27,32-34}$ In this paper, we report the first UK-wide survey of physicians' experiences of adverse events and near misses, and their perceptions of the organisational mechanisms for supporting staff in these circumstances. Until now we have had no knowledge of doctors' experiences or needs in the NHS context, and therefore no information on how to address them. Assumptions are drawn from data in other locations. UK studies published to date are small-scale, conducted at either one or two NHS Trusts, and/or have not included a sample of doctors. ${ }^{35-38}$ This survey of physicians 
in secondary care is particularly significant because they are natural opinion leaders and formers in clinical hierarchies. This group have direct influence on doctors in training, and their attitudes and behaviours may also influence other clinical professionals and have a wider effect on culture within the NHS.

\section{Methods}

We administered an online survey to fellows and members of the Royal College of Physicians (RCP) using their membership database in April 2013. We sent an invitation email containing an embedded link to the survey to all 11,810 physicians for whom valid email addresses were available, with follow-up emails at 2 and 4 weeks. The embedded link led participants to study information and they indicated consent by completing and submitting the anonymous survey. No identifiable information was gathered and surveys were completed confidentially.

We adapted the survey instrument used with US physicians and modified it slightly for UK use. ${ }^{32}$ We used standard definitions for adverse events and near misses (Box 1) and gathered data on respondents' experiences of adverse events, their emotional and behavioural responses to them, and the reporting and disclosure processes for these events. Two items were added to explore the availability of and demand for mentorship. ${ }^{39}$ Seven optional items were also added, which were taken from a validated brief assessment for symptoms of post-traumatic stress disorder (PTSD), to address speculation that PTSD symptoms may arise in these circumstances. No attempt was made, however, to screen or identify individuals at risk of PTSD, which would have been beyond the scope of this work. ${ }^{23,40}$ Face validity checks of the final survey were conducted for relevance, ease of comprehension and ease of use by 10 physician members of the RCP. We made minor amendments, but no changes to validated measures, as a result. NHS ethical approval was not required for this service evaluation exploring the use of incident-reporting systems. Appropriate steps were taken to ensure the anonymity and confidentiality of respondents and this work was conducted in accordance with ethical guidelines for research with human participants. As a membership survey, the study processes and materials were reviewed by the RCP using standard RCP protocols to ensure that the survey conformed to RCP standards. ${ }^{41}$

We first asked respondents if they had ever experienced an adverse event that caused serious patient harm; an adverse event with minor patient harm; a near miss with potential for serious patient harm, or a near miss with potential for minor patient harm. Respondents could cross more than one option. We also provided an option for none of these. Those who had experienced an event were directed to further questions about it. Those who had not were directed to the next section. Similarly for questions related to disclosure, respondents who indicated that they had disclosed an incident were asked further questions about this process, but if not, they were directed on to the next topic.

\section{Results}

The survey link was opened by 1,755 physicians (14.9\% of those on the database), all of whom answered at least one item in the survey. 1,334 answered every item that was relevant to them. A precise response rate is impossible to determine as we do not

\section{Table 1. Demographic information.}

$\begin{array}{lll}\begin{array}{l}\text { Demographic } \\ \text { Years in practice }(n=1,703)\end{array} & \text { \% of respondents } & \mathbf{n} \\ \quad<2 \text { years } & 0.5 & 9 \\ 2-10 \text { years } & 7.6 & 129 \\ >10 \text { years } & 89.7 & 1,527 \\ \text { Retired } & 2.2 & 38 \\ \text { Age }(n=1,668) & & \\ <25 \text { years } & 0.1 & 2 \\ 25-34 \text { years } & 3.5 & 59 \\ 35-44 \text { years } & 32 & 534 \\ 45-54 \text { years } & 37.9 & 632 \\ \text { 55-64 years } & 23.1 & 385 \\ >65 \text { years } & 3.4 & 56 \\ \text { Gender ( } n=1,667) & & 1,037 \\ \text { Male } & 62.2 & 630 \\ \text { Female } & 37.8 & \end{array}$

know how many email recipients saw the survey but made a conscious decision not to participate.

\section{Demographic information}

The mean age of the 1,755 doctors who completed some or all of the survey was 47 years, $37 \%$ were female and $90 \%$ had been in practice for 10 years or more (see Table 1). All (internal) medical specialties were represented. Our sample demographic broadly reflects the profile of members and fellows of the RCP who completed the 2011 census, in which mean age was 48 years and $31 \%$ were female. ${ }^{42}$

\section{Experience of adverse events and near misses}

1,463 respondents $(83.3 \%)$ reported having personally been involved in at least one near miss and/or adverse event at any point in their career (Table 2). Of these, 1,119 (76.5\%) believed that their experience had affected their personal or professional lives. The effects most commonly reported were stress and anxiety, reduction in job satisfaction, difficulties sleeping and loss of professional confidence. Respondents also perceived that the event affected their professional reputation and relationships with colleagues (Table 3 ). Only a small

Table 2. Types of event experienced by participants $(n=1,463)$.

$\begin{array}{lll}\text { Type of event } & \text { \% } & \mathbf{n} \\ \text { Adverse event with serious patient harm } & 51.1 & 748 \\ \text { Adverse event with minor patient harm } & 55.6 & 813 \\ \text { Near miss with potential for serious patient harm } & 61.0 & 892 \\ \text { Near miss with potential for minor patient harm } & 55.1 & 806 \\ \text { None of these } & 10.7 & 157\end{array}$


Table 3. Personal and professional outcomes of an adverse event or a near miss $(n=1,463)$.

\begin{tabular}{lll} 
Outcome & $\%$ & $\mathbf{n}$ \\
Lower confidence in ability as a doctor & 63.2 & 886 \\
Difficulty sleeping & 59.9 & 840 \\
Reduced job satisfaction & 48.5 & 681 \\
Affected relationships with colleagues & 25.5 & 358 \\
$\begin{array}{l}\text { Damaged professional reputation } \\
\text { Other personal or professional outcomes }\end{array}$ & 20.1 & 282 \\
$\begin{array}{l}\text { Anxious about potential for future errors } \\
\text { Generally distressed (eg depressed, upset or }\end{array}$ & 81.5 & 1,192 \\
$\begin{array}{l}\text { angry) } \\
\text { Generally anxious (eg nervous, panicky or tense) }\end{array}$ & 68.0 & 1,077 \\
$\begin{array}{l}\text { Negative towards yourself (eg shame, guilt or } \\
\text { feeling incompetent) }\end{array}$ & 27.3 & 399 \\
$\begin{array}{l}\text { More confident in your abilities (eg feeling } \\
\text { effective, efficient or competent) }\end{array}$ & 7.5 & 110 \\
$\begin{array}{l}\text { Determined to improve (eg feeling determined, } \\
\text { resourceful or strong) }\end{array}$ & 80.6 & 1,179 \\
\hline
\end{tabular}

proportion reported very strong feelings of distress (111; 7.6\%) and/or anxiety $(64 ; 4.4 \%)$. Although negative feelings were unsurprisingly common, respondents were particularly anxious about making errors in the future, and many reported a desire to improve their practice and prevent the recurrence of events as a result (Table 3 ).

\section{Sources of support}

1,313 participants responded to items regarding the sources of support they had used in the past or would like to be available to them after an adverse event; 76 of these $(5.5 \%)$ reported having a formal mentor. 1,142 (87\%) indicated that they would contact a mentor about an adverse event if they had one. Across each age bracket, over $60 \%$ of respondents indicated they would contact a mentor about an adverse event if they had one. The opportunity to contact a mentor was valued the most amongst those with the longest times in practice; $66.1 \%$ of those with over 10 years in practice and $73.7 \%$ of those retired. Respondents also reported that they would speak to peers $(1,116 ; 85 \%)$, family or friends $(869 ; 66.2 \%)$, senior colleagues $(775 ; 59 \%)$, or colleagues from another health profession (399; $30.4 \%) .1,164$ of 1,388 (83.9\%) indicated they had supported a colleague who was affected by an adverse event or near miss. Most $(1,172 ; 66.8 \%)$ did not think that healthcare organisations adequately supported doctors in dealing with the stress associated with an adverse event.

\section{Incident reporting}

Most respondents $(1,141$ of 1,$433 ; 79.6 \%)$ had formally reported an adverse event or near miss using NHS incident-reporting systems; $512(44.9 \%)$ of these were dissatisfied with the way that their report had been dealt with. 364 of 1,463 (25\%) reported that they had been involved in a patient safety incident that
Table 4. Outcomes of reporting an adverse event or near miss $(n=1,141)$.

$\begin{array}{lll}\text { Outcome } & \text { \% } & \text { n } \\ \text { Empathy from colleagues } & 42.7 & 612 \\ \text { Local improvements } & 21.0 & 301 \\ \text { Systems changes } & 19.3 & 277 \\ \text { Useful feedback } & 14.0 & 201 \\ \text { Learning activities } & 8.4 & 120 \\ \text { Closer supervision } & 2.9 & 42 \\ \text { Disciplinary action } & 2.1 & 30 \\ \text { Given more training } & 2.0 & 29 \\ \text { Responsibilities removed } & 1.5 & 21\end{array}$

they hadn't reported, even though they knew they should have done so. Free-text responses revealed beliefs that nothing would improve as a result of making an incident report, that the reporting of errors was an onerous process, and that punitive action was feared. Responses to single items regarding the outcomes experienced as a result of reporting an incident are shown in Table 4.

1,259 of 1,452 respondents $(86.7 \%)$ had disclosed an adverse event or near miss to a patient and/or their family, and most of these $(1,120 ; 89 \%)$ felt satisfied with the way in which they had conducted the disclosure.

\section{Brief PTSD screening measure}

466 respondents (31.9\% female) completed the optional survey items on PTSD symptoms. Of these, 119 (25.5\%) reported symptoms that would be consistent with PTSD. These were broadly representative of the sample in terms of gender, age and time in practice. 49 of these (41.2\%) said they had not reported an incident that should have been reported.

\section{Discussion}

This is the first large-scale UK survey describing the experiences of physicians in relation to adverse patient events. Not surprisingly, most had personally experienced involvement in at least one adverse event and the majority reported being affected either personally or professionally by this. ${ }^{19-21}$ Repercussions for doctors' professional lives were common, including a loss of confidence in their professional ability, reduced job satisfaction and damaged relationships with colleagues. These feelings, coupled with disrupted sleep (reported by over half of the respondents), stress and anxiety could have a direct detrimental effect on patient safety, and might also threaten the development of a strong organisational safety culture in the longer term. ${ }^{32}$ A small number of respondents reported PTSD symptoms. ${ }^{43}$ The personal and professional disruption reported reflects the experiences of trainee doctors and of nurses. Most of our sample were consultant-level physicians and these data suggest that this group have no greater protection from or resilience to such events than more junior colleagues. ${ }^{27-30,35}$ Whilst negative feelings arising after an adverse event were common, $80 \%$ of 
respondents reported a determination to improve following an event and $81.5 \%$ were anxious about the potential for future errors. This suggests that experiencing an adverse event or near miss may actually lead doctors to exercise greater caution.

Most respondents had experience of using formal incidentreporting systems, but only a minority had received useful feedback or seen system changes and improvements as a result. A quarter admitted not reporting an incident even though they knew they should have done so. Our findings reflect previous work in other locations. ${ }^{7-9}$ They suggest that physicians are reluctant to report adverse events because of the personal psychological effects of the incident, a lack of confidence in the incident reporting system, or both. ${ }^{6-9,13}$ Reluctance to report adverse events may be related to a number of factors other than or in addition to these. The NHS (like many other health systems) operates voluntary incident reporting that relies on health professionals' making reports. Incidents that are not reported because health professionals are not satisfied with the process cannot be used for learning and to make changes; therefore, this is an important issue for health care organisations to address.

Few respondents reported access to formal structures within the NHS to support them. This reflects similar findings from North America, where $90 \%$ of physicians reported a lack of adequate organisational support after an event. ${ }^{31,32,34,39}$ Only $5 \%$ had a formal mentor, although most would have found this helpful. In the absence of formal structures, most sought support informally from friends, family and colleagues. These sources have been identified as valued and useful for providing safe and ongoing support, but discussion amongst friends and family may be limited by the need to ensure patient confidentiality. ${ }^{23,26,33}$ Formal mechanisms that assist clinicians in gaining appropriate support might therefore be helpful. Less than $10 \%$ of respondents reported engaging in learning activity or training or being given additional supervision in the aftermath of an incident. The opportunity to learn and make changes after an adverse event has been identified as a strategy valued by clinicians for managing the aftermath of events. ${ }^{31}$

Over $80 \%$ of respondents reported that they were satisfied with their disclosure of an adverse event or near miss to patients and/or families; this is similar to findings in North America but contradicts ${ }^{31}$ patient reports of dissatisfaction with the disclosure process. ${ }^{11,44-46}$ Our findings reinforce the disparity between physicians and patients regarding expectations of incident disclosure, which has been described in US literature as 'the disclosure gap'. ${ }^{11,12}$

\section{Limitations}

Our methodology has limitations. Our findings reflect those of the only other large cross-sectional physician survey on this topic, but a cross-sectional method is reliant on retrospective recall and may explore stable beliefs rather than specific experiences. ${ }^{32}$ Cross-sectional self-reporting also limits the accuracy of information gathered regarding the severity and duration of emotions experienced in relation to an adverse event. Diary methods and longitudinal data may be more informative, although large samples are more difficult to achieve using such methods. Our sample was broadly representative of the RCP Consultant membership, but the use of a responder sample may have shaped the data.
The survey was sent to the email addresses of 11,810 members and fellows of the RCP and therefore is limited by a very low response rate. We lack knowledge of the true response rate as it is impossible for us to determine if all emails reached the intended recipients; some may have been diverted by spam filters or firewalls. Low response rates are typical of other recently conducted UK consultant membership surveys. ${ }^{42,47}$ A number of factors may have influenced the response rate, including the use of an online survey over a short time period, limited reminders, the sensitive nature of this topic, lack of incentive, and the respondents relationship with or perception of the RCP. ${ }^{48}$ Those at either extreme who were strongly affected or not affected at all by their experience may not have been inclined to participate. We do not know the extent to which physicians' experiences reflect those from other specialties, such as surgery, or those of physicians at other levels of training. Respondents were included from each of the four UK systems that operate different systems for incident reporting. Although reporting principles are the same in each of these systems, comparisons between the experiences of doctors using each of the four systems was impossible because we did not capture details of the system that each respondent used. These data provide a unique insight into the experiences of UK physicians, but lack of comparable UK data means that we cannot comment on the reliability of these findings.

\section{Implications}

Our findings provide evidence that many NHS consultant physicians do not feel confident and safe in reporting adverse events or supported in effectively managing their experiences of such events. It is unlikely that current incident-reporting systems, or the introduction of a legal duty of candour, will improve patient safety until the NHS recognises and addresses these issues. ${ }^{49,50}$ Many factors, including some of those that we have described, make clinicians reluctant to report incidents or to discuss them fully and openly with patients. The current debate in the NHS in England, arising from the Francis report, on a legally enforceable duty of candour (to oblige clinicians to disclose details of incidents to patients), does not seem to have considered any of the more complex reasons behind low reporting rates. It seems unlikely that a legal duty of candour will be effective without considering some of these issues. These findings are significant given that similar models of incident reporting and policies for the disclosure of incidents to patients and carers (in which consultant physicians play a crucial role) operate in many countries. ${ }^{38}$ As consultant physicians have a strong influence on healthcare culture, their negative experiences are also likely to impact those that they supervise and clinicians from other disciplines. Healthcare organisations, commissioners, policy makers, regulators and professional bodies have a responsibility to develop systems to support clinicians who have been affected by these experiences in order to foster the open, transparent culture that is necessary and to ensure that incident reporting truly becomes a learning activity.

Substantial gaps in the literature in this area remain, and more UK data are needed to qualify our findings. Longitudinal data are lacking internationally and will be crucial in establishing both the duration over which clinicians are affected and how the effects of an adverse event change (if at all) over time. Further work may seek to establish the factors (such as specialty, time in practice and seniority) associated 
with particular reactions and the more (and less) successful strategies for the management of adverse events and near misses. In particular, there may be value in determining the factors that drive clinicians' determination to improve. More rigorous evaluation of current models of support is also needed to determine the effectiveness of these approaches. ${ }^{34} \mathrm{~A}$ small number of respondents reported symptoms suggestive of PTSD. More detailed accounts of the experiences of this group may provide insight into particular circumstances that give rise to a more severe response.

\section{Acknowledgements}

We are grateful to the president, other senior officers and the External Affairs team at the RCP for supporting and publicising our work and to the RCP fellows and members who generously responded to the survey. Nina Newbury and colleagues in the RCP Workforce Unit kindly advised on and supported our questionnaire. Colleagues in the RCP and beyond provided helpful feedback on the questionnaire and on early drafts of the manuscript, for which we are grateful.

\section{References}

1 Department of Health, The Rt Hon Jeremy Hunt MP. The silent scandal of patient safety (Speech). Delivered on 21 June 2013. Available online at www.gov.uk/government/speeches/the-silentscandal-of-patient-safety [Accessed 10 November 2014].

2 Francis R. Report of the Mid Staffordshire NHS Foundation Trust Public Inquiry. London: Stationary Box, 2013.

3 Williams SK, Osborn SS. The development of the National Reporting and Learning System in England and Wales, 2001-2005. Med J Aust 2006;184:S65-8.

4 Runciman WB, Williamson JAH, Deakin A et al. An integrated framework for safety, quality and risk management: an information and incident management system based on a universal patient safety classification. Qual Saf Health Care 2006;15:182-90.

5 Kohn LT, Corrigan JM, Donaldson MS (Eds). To err is human: building a safer health system. Washington, DC: National Academy Press, 1999:156.

6 Lawton RJ, Parker D. Barriers to reporting incidents in a health care system. Qual Saf Health Care 2002;11:15-8.

7 Evans SM, Berry JG, Smith BJ et al. Attitudes and barriers to incident reporting: a collaborative hospital study. Qual Saf Health Care 2006;15:39-43.

8 Kingston MJ, Evans SM, Smith BJ et al. Attitudes of doctors and nurses towards incident reporting: a qualitative analysis. Med J Aust 2004;18:136-9.

9 Rowin EJ, Lucier D, Pauker SG et al. Does error and adverse event reporting by physicians and nurses differ? Jt Comm J Qual Pat Saf 2008;34:537-45.

10 Nuckols TK, Bell DS, Liu H et al. Rates and types of events reported to established incident reporting systems in two US hospitals. Qual Saf Health Care 2007;16:164-8.

11 Gallagher TH, Waterman AD, Ebers AG et al. Patients' and physicians' attitudes regarding the disclosure of medical errors. JAMA 2003;289:1001-7.

12 Gallagher TH, Garbutt JM, Waterman AD et al. Choosing your words carefully: how physicians would disclose harmful medical errors to patients. Arch Intern Med 2006;166:1585-93.

13 Jennings PA, Stella J. Barriers to incident notification in a regional pre-hospital setting. Emerg Med J 2011;28:526-9.

14 Waring J. Beyond blame: the cultural barriers to medical incident reporting. Soc Sci Med 2005;60:1927-35.

15 Wu AW, Cavanaugh TA, McPhee SJ et al. To tell the truth: ethical and practical issues in disclosing medical mistakes to patients.

J Gen Intern Med 1997;12:770-5.
16 Kaldjian LC, Jones EW, Rosenthal GE et al. An empirically derived taxonomy of factors affecting physicians' willingness to disclose medical errors. J Gen Intern Med 2006;21:942-8.

17 Robbennolt, J. Apologies and medical error. Clin Orthop Relat Res 2009;467:376-82.

18 Sirriyeh R, Lawton RJ, Gardner P et al. Coping with medical error: a systematic review of papers to assess the effects of involvement in medical error on health care professional's psychological wellbeing. Qual Saf Health Care 2010;19:1-8.

19 Schwappach DLB, Boluarte TA. The emotional impact of medical error involvement on physicians: a call for leadership and organisational accountability. Swiss Med Wkly 2009;139:9-15.

20 Seys D, Scott S, Wu AW et al. Supporting involved health professional (second victims) following an adverse event: a literature review. Int J Nurs Stud 2013;50:678-87.

21 Wu AW. Medical error: the second victim. BMJ 2000;320:726.

$22 \mathrm{Wu}$ AW, Steckler R. Medical error, incident investigation and the second victim: doing better but feeling worse? BMJ Qual Saf 2012;21:267-70.

23 Dekker, S. Second victim: error, guilt, trauma, and resilience. Boca Raton, FL: CRC Press, 2013.

24 Christensen JF, Levinson W, Dunn PM. The heart of darkness - the impact of perceived mistakes on physicians. J Gen Intern Med 1992;7:424-31.

25 Mizrahi T. Managing medical mistakes: ideology, insularity and accountability among internists-in-training. Soc Sci Med 1984;19;135-46.

26 Scott SD, Hirschinger LE, Cox KR et al. The natural history of recovery for the health care provider 'second victim' after adverse patient events. Qual Saf Health Care 2009;18:325-30.

$27 \mathrm{Wu}$ AW, Folkman S, McPhee SJ et al. How house officers cope with their mistakes. West J Med 1993;159:565-9.

28 Wu AW, Folkman S, McPhee SJ et al. Do house officers learn from their mistakes? Qual Saf Health Care 2003;12:221-7.

29 West CP, Mashele M, Huschka MM et al. Association of perceived medical errors with resident distress and empathy — a prospective longitudinal study. JAMA 2006;296:1071-8.

30 Muller D, Ornstein K. Perceptions of and attitudes towards medical errors among medical trainees. Med Educ 2007;41:645-52.

31 Harrison R, Lawton R, Perlo J et al. Emotion and coping in the aftermath of error: a cross country exploration. J Pat Saf 2013, in press.

32 Waterman AD, Garbutt J, Hazel E et al. The emotional impact of medical errors on practicing physicians in the United States and Canada. Jt Comm J Qual Patient Saf 2007;33:467-76.

33 Hu Y, Fix M, Hevelone ND et al. Physicians needs in coping with emotional stressors: the case for peer support. Arch Surg 2012;147:212-7.

34 Scott S, Hirschinger L, Cox K et al. Caring for our own: deploying a system wide second victim rapid response team. Jt Comm J Qual Patient Saf 2010;36:233-40.

35 Meurier CE, Vincent CA, Parmar DG. Learning from errors in nursing practice. J Adv Nurs 1997;26:111-9.

36 Pinto A, Faiz O, Vincent C. Managing the after effects of serious patient safety incidents in the NHS: an online survey study. BMJ Qual Saf 2012;21:1001-8.

37 Pinto A, Faiz O, Bicknell C et al. Surgical complications and their implications for surgeons' well-being. Br J Surg 2013;100:1748-55.

38 World Alliance for Patient Safety (WHO). WHO draft guidelines for adverse event reporting and learning systems: from information to action. Geneva: WHO Publications, 2005.

39 Harrison R, Mcclean S, Lawton R, Wright J, Kay C. Mentorship for newly appointed consultants: a strategy for enhancing patient safety? J Patient Saf 2014;10:159-67.

40 Bohnert KM, Breslau N. Assessing the performance of the short screening scale for post traumatic stress disorder in a large nationally representative survey. Int J Methods Psychiatr Res 2011;20:e1-5. 
41 World Medical Association, Inc. WMA declaration of Helsinki - ethical principles for medical research involving human subjects. Available online at www.wma.net/en/30publications/10policies/b3/ index.html [Accessed 22 August 2013].

42 Federation of the Royal Colleges of Physicians of the UK. Census of consultant physicians and medical registrars in the UK 2011. Royal College of Physicians, London, 2013. Available online at www.rcplondon.ac.uk/sites/default/files/2011_census_-_registrar_ census_-_intro_and_r1-r20.pdf [Accessed 10 November 2014]

43 Breslau N, Peterson EL, Kessler RC et al. Short screening scale for DSM-IV posttraumatic stress disorder. Am J Psychiatry 1999;156:908-11.

44 O'Conner E, Coates HM, Yardley IE et al. Disclosure of patient safety incidents: a comprehensive review. Int J Qual Health Care 2010;22:371-9.

45 Iedema R, Allen S, Britton K et al. Patients' and family members' views on how clinicians enact and how they should enact incident disclosure: the "100 patient stories" qualitative study. BMJ 2011;343:d4423.

46 Iedema R, Allen S, Sorensen R et al. What prevents incident disclosure, and what can be done to promote it? Jt Comm J Qual Patient Saf 2011;37:409-17.
47 Medical Protection Society, 2013. Eight in ten doctors are concerned online medical records will increase workload, MPS survey reveals. Available online at www.medicalprotection.org/uk/press-releases/ Eight-in-ten-doctors-are-concerned-online-medical-records-willincrease-workload-MPS-survey-reveals [Accessed 10 November 2014].

48 Dykema J, Jones NR, Piché T et al. Surveying clinicians by web current issues in design and administration. Eval Health Prof 2013;36:352-81.

49 Department of Health, 2007. Building a safer NHS: implementing an organisation with a memory. London: National Academy, 2001.

50 Department of Health, 2011. Implementing a 'Duty of Candour'; a new contractual requirement on providers. Proposals for consultation. Available online at http://webarchive.nationalarchives.gov.uk/+/www. dh.gov.uk/en/Consultations/Liveconsultations/DH_130400 [Accessed 6 August 2014].

Address for correspondence: Dr R Harrison, 314 Edward Ford Building, School of Public Health, Sydney Medical School, University of Sydney, Sydney, NSW 2006, Australia. Email: reema.harrison@sydney.edu.au

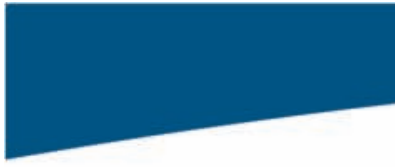

Join the discussion online

in $6 \frac{\text { You }}{\text { Toube }} \in$

www.linkedin.com/company/royal-college-of-physicians www.twitter.com/rcplondon www.youtube.com/rcponline www.facebook.com/royalcollegeofphysicians

$$
\text { Physicians }
$$

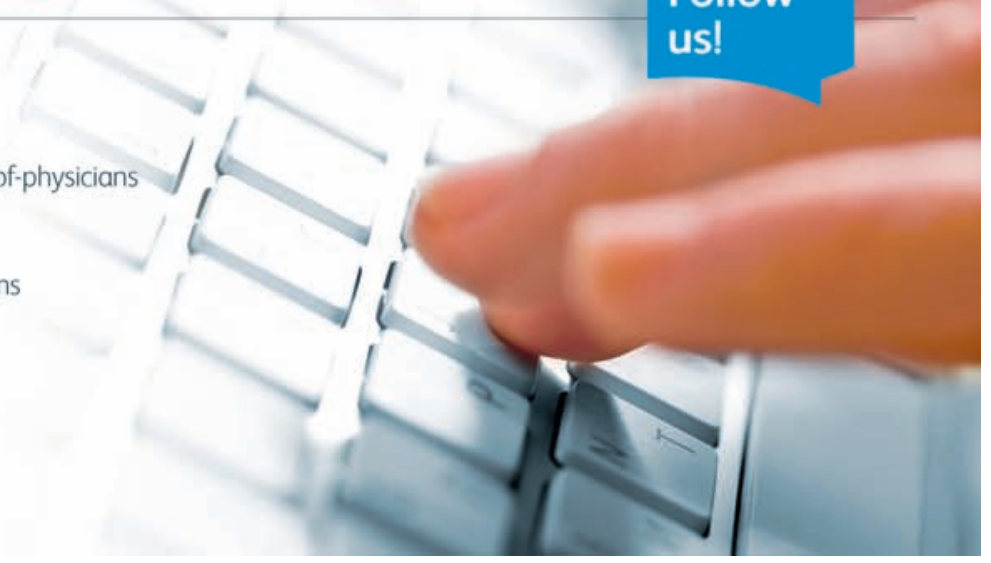

